\title{
Focal hand dystonia in a patient with thoracic outlet syndrome
}

\author{
A Quartarone, P Girlanda, G Risitano, G Picciolo, S Sinicropi, C Nicolosi, V Macaione, \\ C Messina
}

\begin{abstract}
A patient affected by thoracic outlet syndrome, with an involvement of the left lower primary trunk due to a rudimentary cervical rib, developed a severe hand dystonia on the same side. The dystonic posture was characterised by a flexion of the wrist with the fingers curled into the palm. Polygraphic recordings performed on the left flexor digitorum superficialis (FDS4) and extensor digitorum superficialis (EDC4) muscles, during a repetitive tapping task of the fourth digit, showed a loss of well formed bursts without a clear silent period along with long duration bursts of cocontraction in antagonistic muscles. The study of reciprocal inhibition between forearm flexor and extensor muscles showed a reduced amount of inhibition in both the disynaptic and the later presynaptic phase of inhibition. The patient underwent an operation with resection of the cervical rib. Twelve hours after the operation the patient experienced a significant improvement of the hand dystonia; the distonia had disappeared completely by two months with a progressive normalisation of reciprocal inhibition.

(f Neurol Neurosurg Psychiatry 1998;65:272-274)
\end{abstract}

Keywords: thoracic outlet syndrome; focal dystonia; reciprocal inhibition cocontraction

An increasing number of reports have focused attention on the importance of trauma as a cause or precipitant factor of movement disorders. It has now been well established that trauma to the CNS can cause Parkinson's disease, tremor, and dystonia. ${ }^{12}$ On the contrary the effect a peripheral nervous system disease has on the pathogenesis of movement disorders such as myoclonus, ${ }^{3}$ painful legs and moving toes disease, ${ }^{4}$ and Parkinson's disease ${ }^{5}$ is less clear. More recently, there have been numerous papers describing the association of soft tissue injury, mononeuropathy, radiculopathy, and plexopathy with focal dystonia. ${ }^{67} \mathrm{~A}$ high incidence of ulnar neuropathy has been found in musician's cramp. In these patients the focal dystonia improves and worsens in parallel with fluctuations of ulnar neuropathy. ${ }^{8}$ This report is on a patient affected by a plexopathy due to a thoracic outlet syndrome, who developed a severe focal hand dystonia on the same side, which improved dramatically after operation.

\section{Case report}

We describe a young man who had been complaining of intermittent pain and paraesthesias along the medial aspect of the left arm and forearm for the past two years. In the past six months, he has also experienced numbness of the fourth and fifth digits of the left hand, associated with hand weakness and difficulty in the performance of flexion and extension movements of the wrist. Neurological examination disclosed a mild wasting of hypothenar muscles associated with a dystonic posture of the left hand characterised by a flexion of the wrist and curling of the fingers into the palm. A patchy sensory loss was detected along the medial aspect of the left forearm extending into the fourth and fifth digit. In particular, vibration sense, joint position, and two point discrimination was lost. Neck radiography disclosed the presence of a bilateral rudimentary cervical rib as well as a bilateral elongated transverse process of C7.The Addson test was negative. Arteriography showed only a mild spasm of the subclavian artery. Electroneurographic studies disclosed a reduced median nerve compound motor action potential (CMAP) recorded from the abductor pollicis brevis, a reduced sensory nerve action potential (SNAP) recorded from the ulnar nerve, and a prolonged latency of $F$ wave in hypothenar muscles on the left side. Conduction velocity reduction was not detected along the ulnar nerve at the elbow. Concentric needle EMG suggested chronic partial denervation in the muscles supplied from C8 and T1. The somatosensory evoked potentials, after stimulation of the ulnar nerve, showed an increased N9-N13 interpeak conduction time after stimulation on the left side. Gadolinium enhanced MRI of the brachial plexus and cervical spinal cord was normal. Polygraphic surface recording performed on the left flexor digitorum superficialis (FDS4) and extensor digitorum superficialis (EDC4) muscles, during a repetitive tapping task of the fourth digit, demonstrated a loss of well formed bursts without a clear silent period along with long duration bursts of cocontraction in antagonistic muscles 

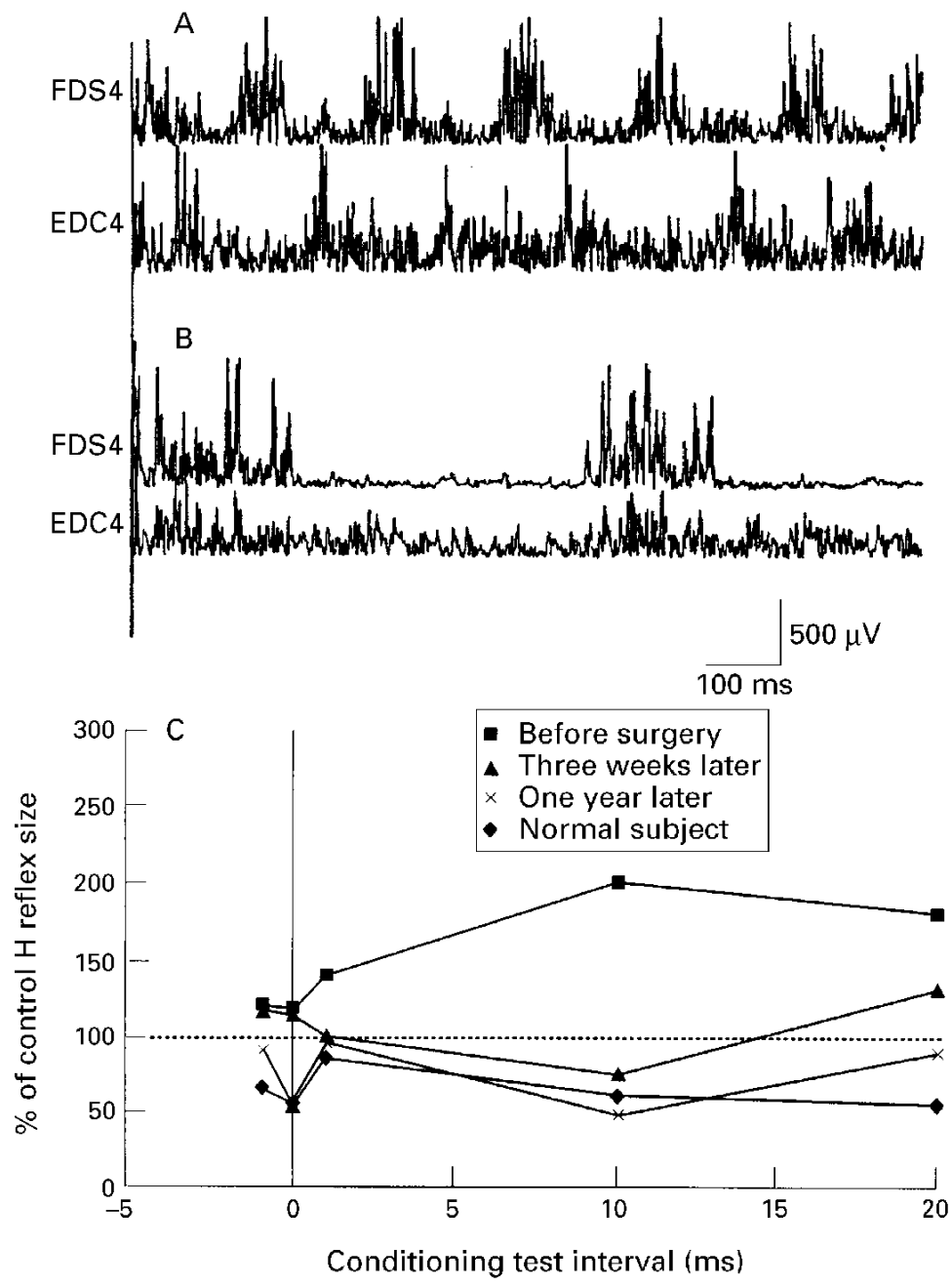

(A) Representative samples of burst patterns in a normal subject, and (B) in our patient affected by the hand dystonia. (C) Time course of $H$ reflex inhibition in the wrist flexors after single motor threshold conditioning shock given to the radial nerve in the spiral groove. Each point is the mean of 10 trials. The abscissa plot the timings of the median nerve test shock relative to that of radial nerve conditioning shock which was given at time 0 ms. Negative timings indicate that median nerve shock occurred first. The size of the conditioned $H$ reflex was expressed as a percentage of the size of 10 control unconditioned trials $(100 \%$ is plotted in thedotted line). The different curves plot the reciprocal inhibition in our patient before the surgical operation, 3 weeks later, and after 1 year, compared with a normal subject.

(figure B). The study of reciprocal inhibition between forearm flexor and extensor muscles, when compared with a population of 10 normal subjects, showed a reduced amount of inhibition in both the disynaptic and the later presynaptic phase of inhibition. The details of this technique are given elsewhere. ${ }^{9}$ The patient underwent a surgical operation with resection of the cervical rib. Twelve hours after the operation the patient experienced relief of pain and considerable improvement of the hand dystonia; the distonia had disappeared completely by two months. We checked the reciprocal inhibition after the operation and over the subsequent months (figure C), noting a progressive normalisation of both phases of inhibition.

\section{Discussion}

The electrophysiological findings were consistent with the diagnosis of thoracic outlet syndrome with an involvement of the primary low trunk of the left brachial plexus. The patient also presented a dystonic posture of the left hand with loss of speed and fluency of movement, which improved dramatically after surgical operation. The activity in FDS4 and in EDC4 muscles recorded via surface electrodes after a tapping task of the fourth digit disclosed a dystonic cocontraction pattern that was confirmed by the reciprocal inhibition technique. This report shows that the peripheral nervous system could be involved in the determinism of focal hand dystonia. This is supported by the fact that surgical operation relieved the hand dystonia with a progressive normalisation of the reciprocal inhibition. The notion that peripheral nervous system involvement alters sensory input, inducing central cortical and subcortical reorganisation, generating movement disorders, has received support from recent experimental studies. Sanes et $a l^{10}$ found a reorganisation of cortical motor outputs from the shoulder area after forelimb amputation in adult rats. Merzenich et $a l^{11}$ discovered that median nerve section in adult monkeys rendered the area of median nerve cutaneous representation in the sensory cortex responsive to radial and ulnar stimulation. Plasticity of the motor cortex has also been reported in humans after amputation or anaesthetic block. ${ }^{12}$ However, the mechanism underlying CNS plasticity after peripheral nerve injury is still open to speculation. Focal hand dystonia is characterised by a reduction in ipsilateral reciprocal inhibition due to a dysfunction of interneurons responsible for such inhibition at the spinal level. ${ }^{13}$ Activity in these interneurons is regulated by cortical control and by the peripheral input from I $\alpha$ afferents. Stimulation of I $\alpha$ afferents, using a high frequency vibration applied to the tendon and muscle belly, partially reproduces the symptoms in patients with writer's cramp. ${ }^{14}$ These data suggest that focal dystonia is influenced by tactile and propioceptive sensory inputs. The findings obtained in our patient agreed with the data described in the literature. We hypothesise that a focal demyelination within the brachial plexus of our patient produced an ephaptic activation of cutaneous and muscle afferents. We maintain that the chronic activation of cutaneous and I $\alpha$ afferents may alter reciprocal inhibition at a spinal level, thus inducing EMG abnormalities similar to those seen in dystonia. The surgical operation may have been responsible for breaking this vicious circle, thereby relieving the hand dystonia.

1 Factor S, Sanchez Ramen J, Weiner WJ. Trauma as an etiology of Parkinsonism. A historical review of the concept. Mov Disord 1988;3:30-7.

2 Obeso JA, Norbone J. Post-traumatic tremor and myoclonic jerking. I Neurol Neurosurg Psychiatry 1983;46:488.

3 Jankovic J, Pardo R. Segmental myoclonus: clinical and pharmacologic slides. Arch Neurol 1986;43:1025-31.

4 Dressler D, Thompson PD, Gledhill RF, et al. The syndrome of painful legs and moving toes. Mov Disord 1994;9:13-21.

5 Walker GF. Parkinsonism following peripheral trauma. BMf 1937;2:65.

6 Jankovic J, Van Der Linden C. Dystonia and tremor induced by a peripheral trauma: predisposing factors. $\mathcal{F}$ Neurol Neuby a peripheral trauma: predisposing
rosurg Psychiatry 1988;51:1512-9.

7 Schott GD. Induction of involuntary movements by peripheral trauma: an analogy with causalgia. Lancet 1986;27: 712-6. 
8 Charness ME. The relationship between local injury and focal dystonias in performing artists. Neurology 1989: 39(suppl 1):246-7.

9 Day BL, Marsden CD, Obeso JA, et al. Reciprocal inhibition between the muscles of the human forearm. F Physiol 1984; 349:519-34.

10 Sanes J, Suner S, Donoghue J. Dynamic organisation of primary motor cortex output to target muscles in adult rats. Long term patterns of reorganisation following motor or mixed nerve lesions. Exp Brain Res 1990;79:479-91.

11 Merzenich MM, Kaas JH, Wall JT, et al. Progression of change following median nerve section in the cortical representation of the hand in areas $3 \mathrm{~b}$ and 1 in adult owl and squirrel monkeys. Neuroscience 1983;10:639-65.

12 Brasil-Neto JP, Cohen LG, Pascual Leone A, et al. Rapid reversible modulation of human motor output after transient deafferentation of the forearm: a study with transcranial magnetic stimulation. Neurology 1992;42:1302-6.

13 Panizza ME, Hallett M, Nilsson J. Reciprocal inhibition in patients with hand cramps. Neurology 1989;39:85-9.

14 Kaji R, Rothwell JC, Katayama M, et al. Tonic vibration reflex and muscle afferent block in writer's cramp. Ann Neurol 1995;38:155-62. 